\title{
Supplement to "Attribution of precipitation to cyclones and fronts over Europe in a kilometer-scale regional climate simulation”
}

Stefan Rüdisühli ${ }^{1}$, Michael Sprenger ${ }^{1}$, David Leutwyler ${ }^{2}$, Christoph Schär $^{1}$, and Heini Wernli ${ }^{1}$

${ }^{1}$ Institute for Atmospheric and Climate Science, ETH Zurich, Switzerland

${ }^{2}$ Max Planck Institute for Meteorology, Hamburg, Germany

Correspondence: Stefan Rüdisühli (stefan.ruedisuehli@env.ethz.ch) 
winter

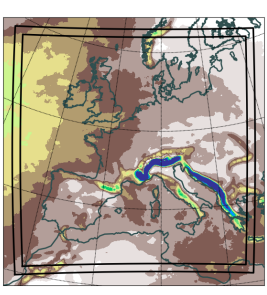

(a-d) cold fronts

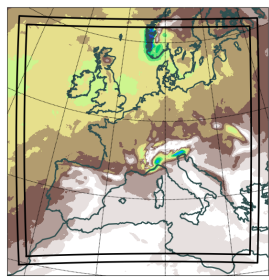

(e-h) warm fronts

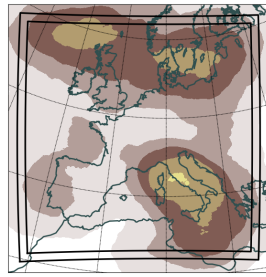

(i-I) cyclones

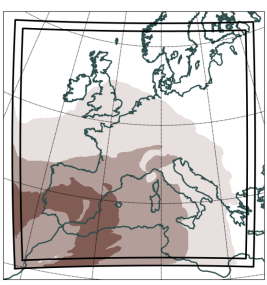

$(\mathrm{m}-\mathrm{p})$ high pressure spring
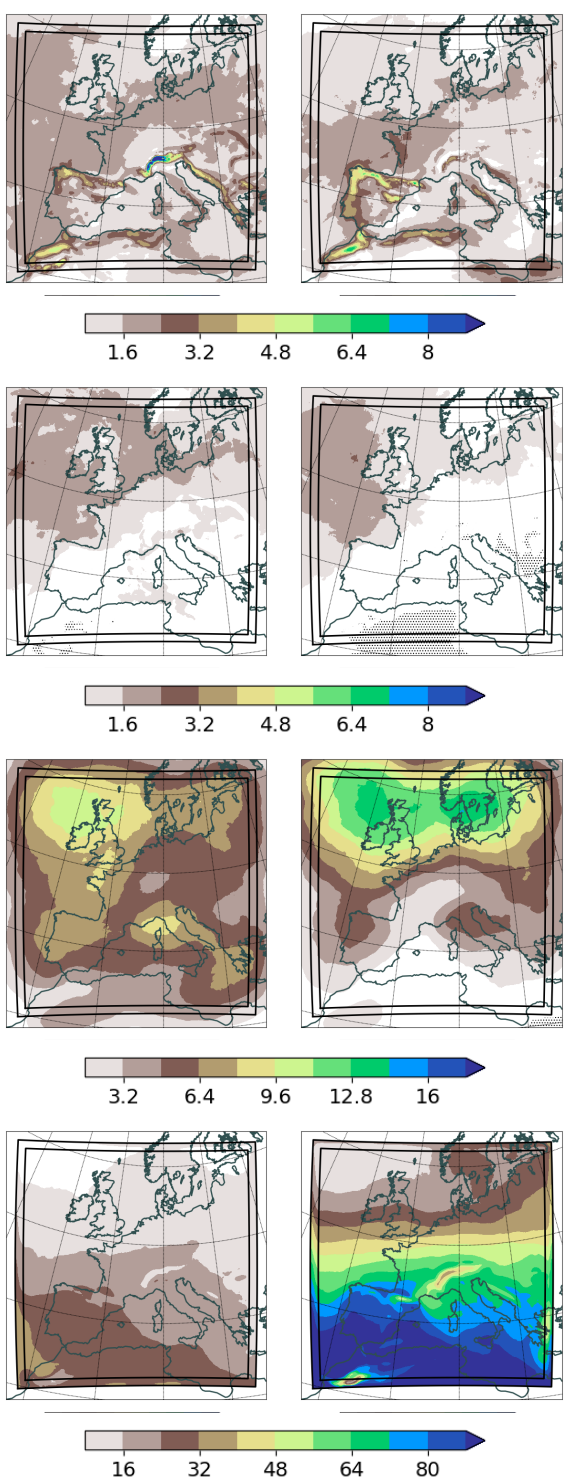

fall

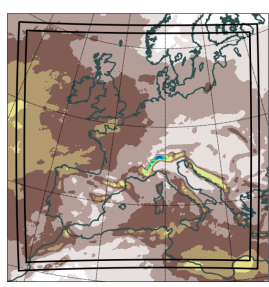

feature frequency ( $\%)$

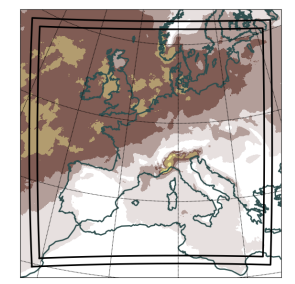

feature frequency $(\%)$

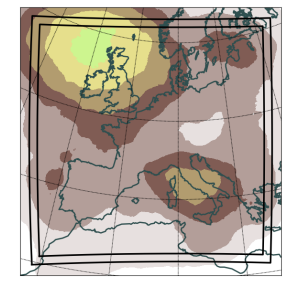

feature frequency $(\%)$

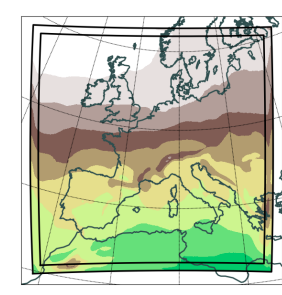

feature frequency ( $\%)$

Figure S1. Feature frequencies of (a-d) cold fronts, (e-h) warm fronts, (i-l) cyclones, and (m-p) high-pressure areas during (left to right) winter, spring, summer, and fall 2000-2008. The outer black box shows the computational domain of the $2.2 \mathrm{~km}$ simulation, the inner box the analysis domain. The fields are computed by first reducing each feature in the respective time period to a binary mask field, and then averaging these binary fields to obtain the total feature frequency field. 


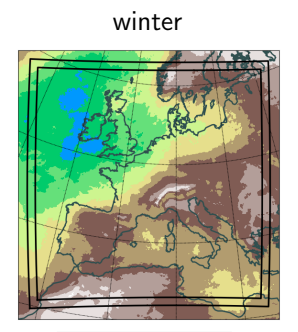

(a-d) cold fronts

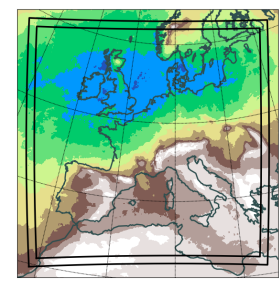

$(\mathrm{e}-\mathrm{h})$ warm fronts

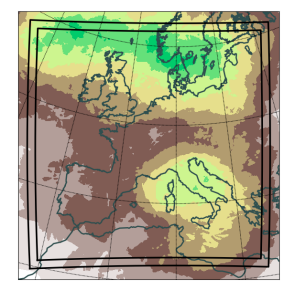

(i-I) cyclones
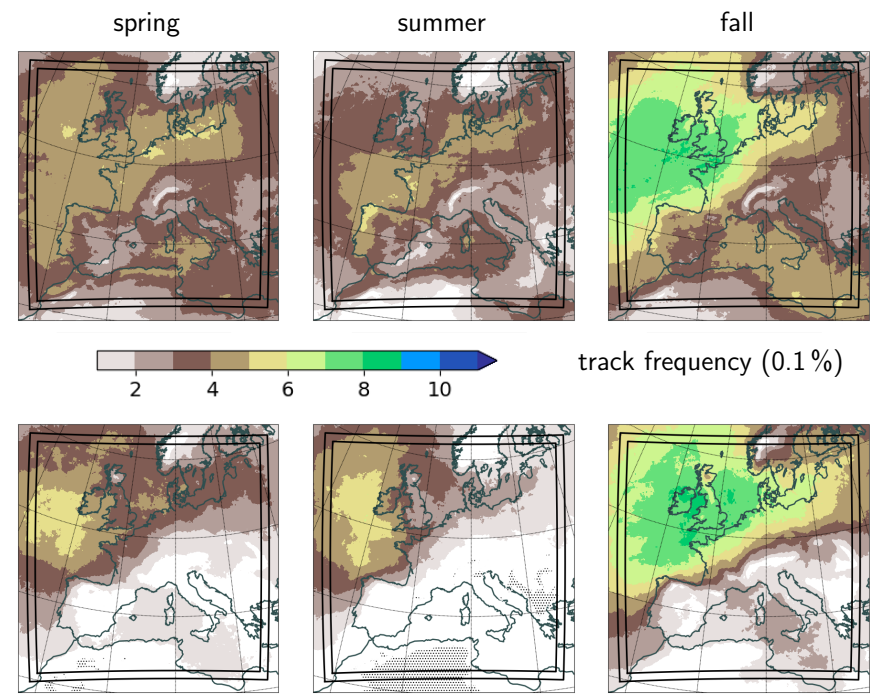

track frequency $(0.1 \%)$
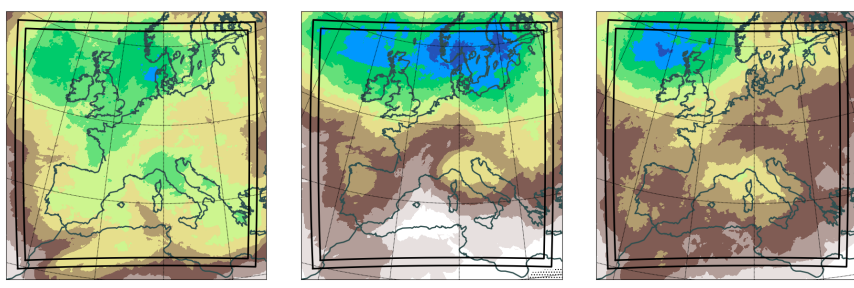

track frequency $(0.1 \%)$

Figure S2. Track frequencies of (a-d) cold fronts, (e-h) warm fronts, and (i-l) cyclones during (left to right) winter, spring, summer, and fall 2000-2008. The outer black box shows the computational domain of the $2.2 \mathrm{~km}$ simulation, the inner box the analysis domain. The fields are computed by first reducing each track to a binary mask field comprised of all grid points affected by any feature belonging to the track in the respective time period, and then averaging these binary fields to obtain the total track frequency field. 


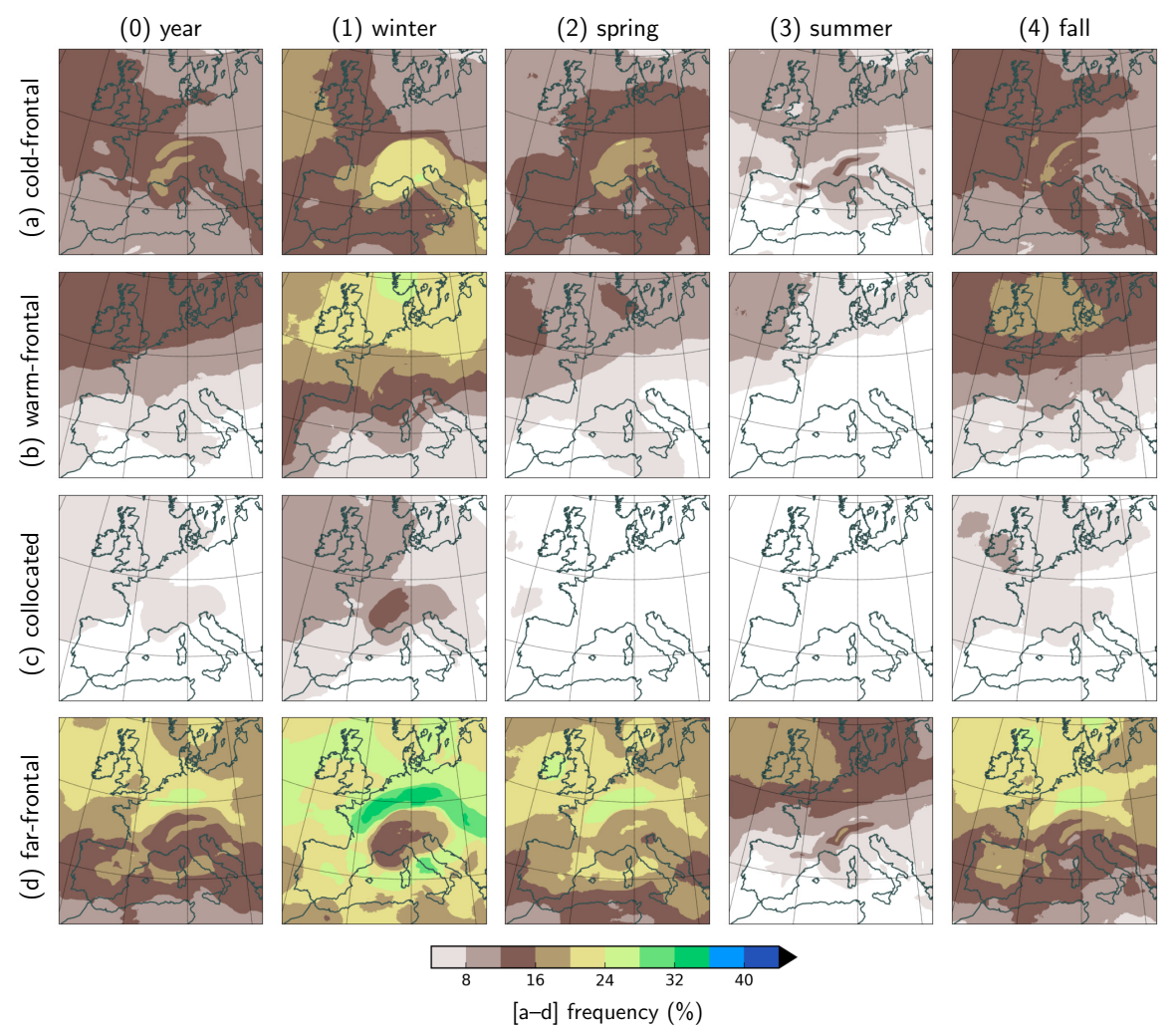

Figure S3. Frequencies of front-cyclone-relative components during (0) the whole year, (1) winter (DJF), (2) spring (MAM), (3) summer (JJA), and (4) fall (SON) 2000-2008. Shown are the (a) cold-frontal, (b) warm-frontal, (c) collocated, and (d) far-frontal components. 


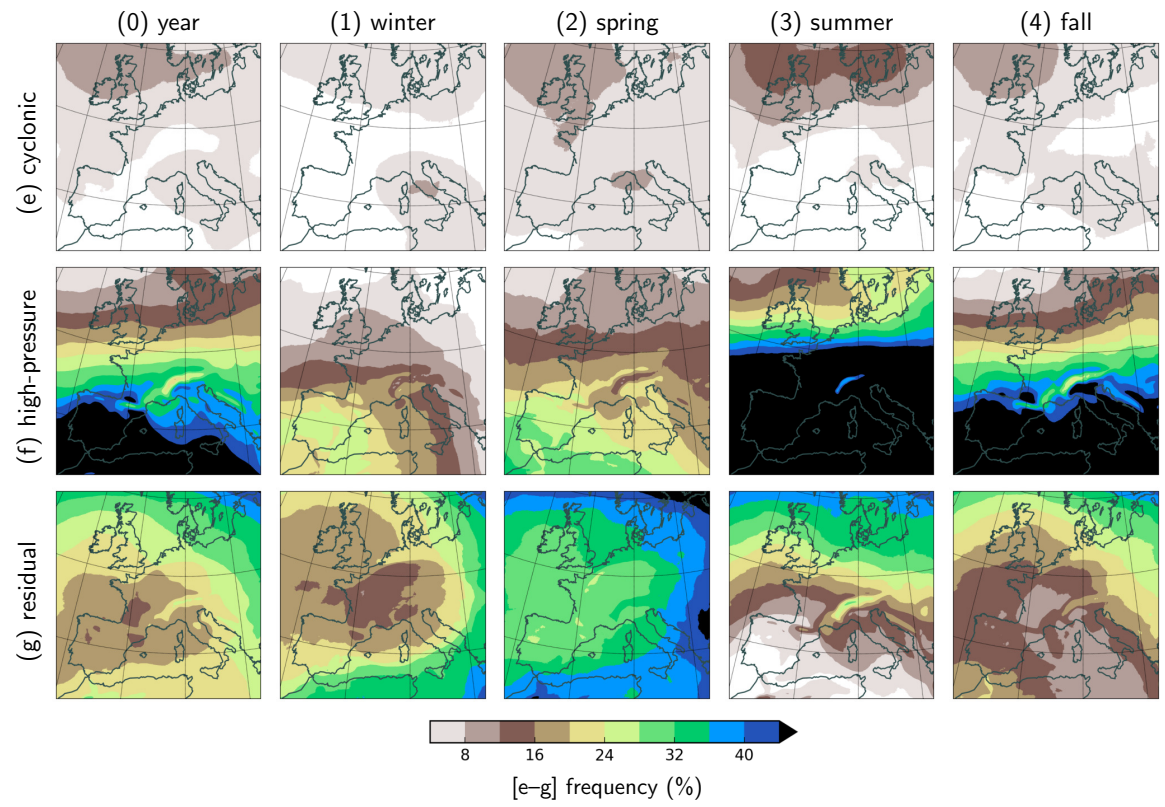

Figure S4. Like Fig. S3, but showing the frequencies of the (e) cyclonic, (f) high-pressure, and (g) residual components. 

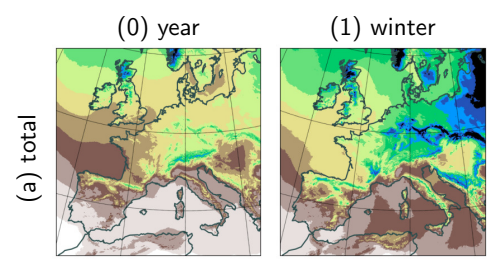

(2) spring
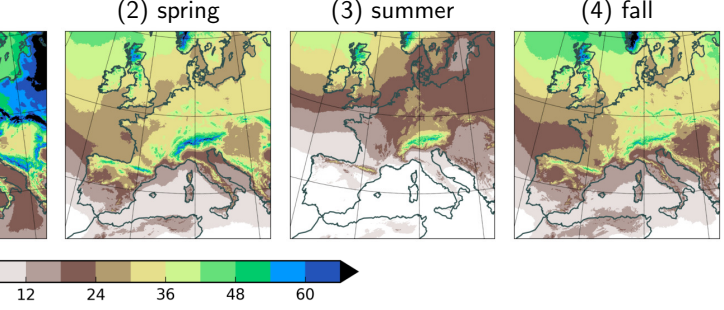

[a] wet hour frequency $(\%)$
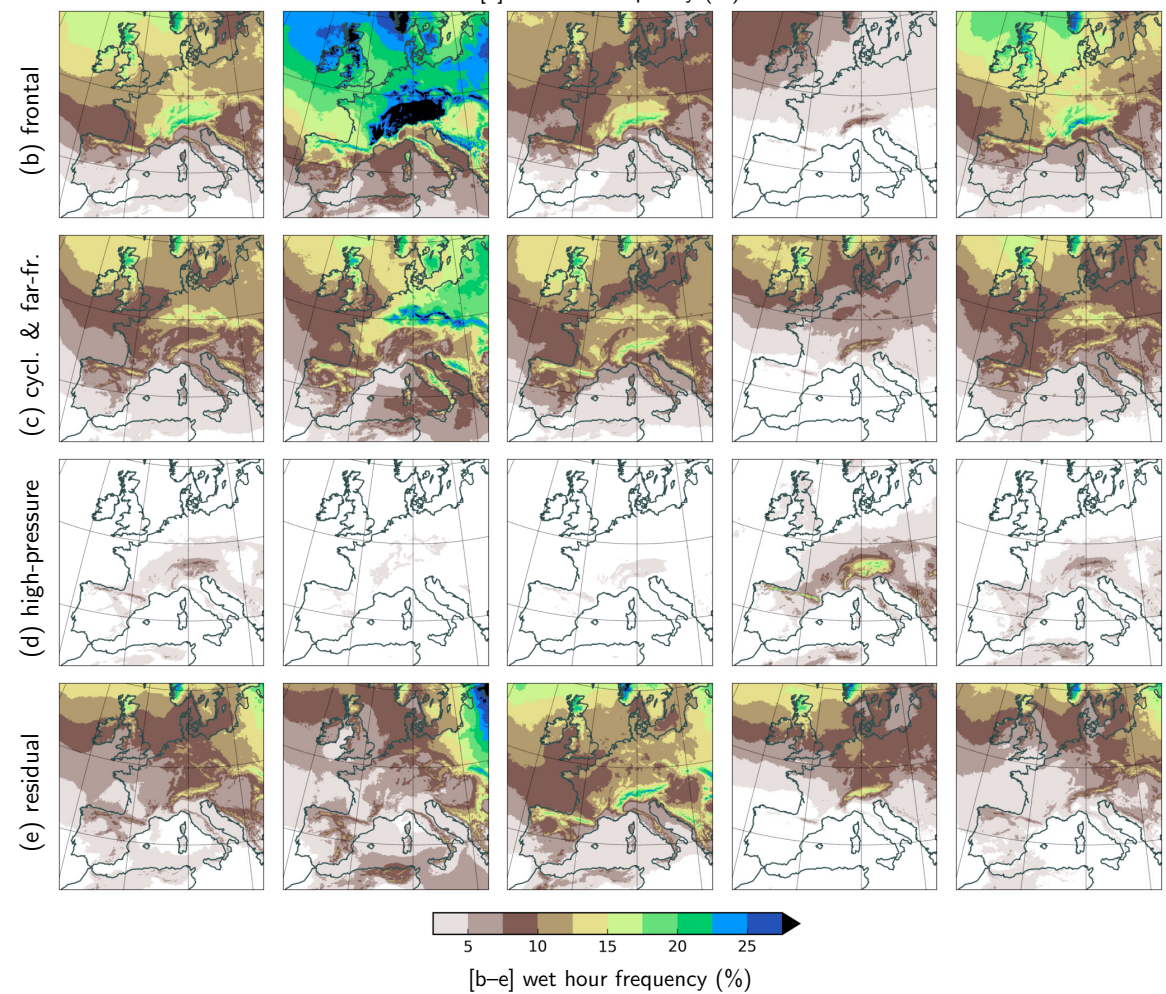

Figure S5. Wet-hour frequency during (0) the whole year, (1) winter (DJF), (2) spring (MAM), (3) summer (JJA), and (4) fall (SON) 20002008, (a) overall and (b-e) for sets of front-cyclone-relative components, specifically: (b) sum of cold-frontal, warm-frontal, and collocated; (c) sum of cyclonic and far-frontal; (d) high-pressure; and (e) residual. 
(0) year
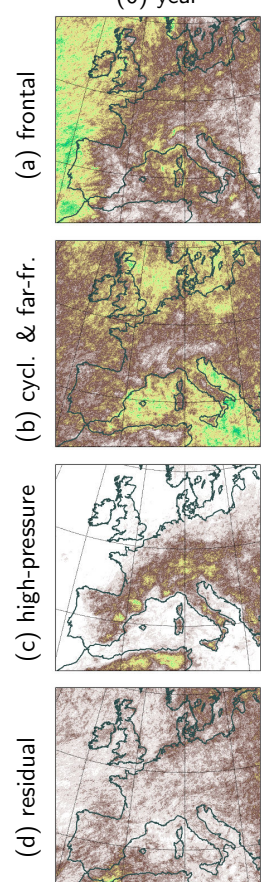

(1) winter
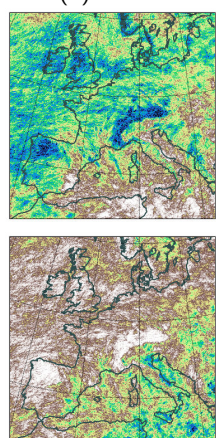
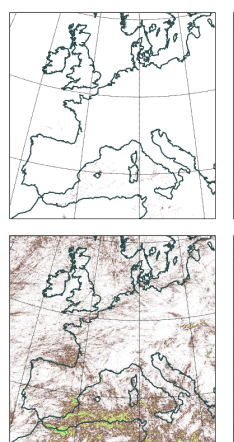

(3) summer
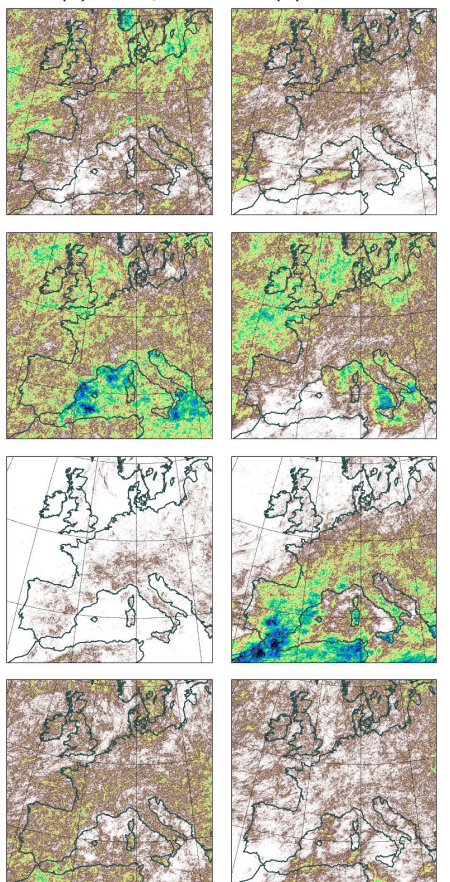

$x^{2}+x^{2}$
(4) fall
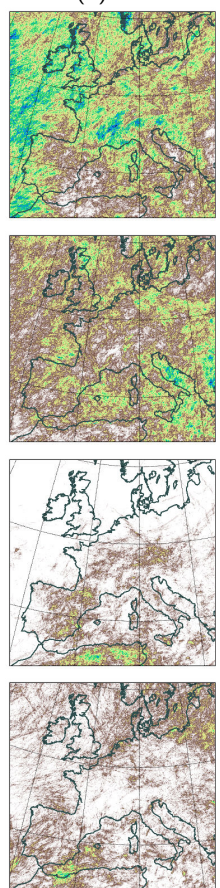

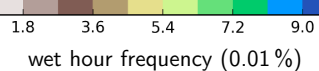

Figure S6. Like Fig. S5 b-d but for heavy precipitation, showing the frequency of hours with precipitation exceeding the local $99.9^{\text {th }}$ all-hour percentile of hourly precipitation. 